\title{
Role of microRNAs in progenitor cell commitment and osteogenic differentiation in health and disease (Review)
}

\author{
MARIA TERESA VALENTI ${ }^{1}$, LUCA DALLE CARBONARE ${ }^{1}$ and MONICA MOTTES ${ }^{2}$ \\ Departments of ${ }^{1}$ Medicine and ${ }^{2}$ Neurosciences, Biomedicine and Movement Sciences, \\ Biology and Genetics Section, University of Verona, Verona I-37134, Italy
}

Received July 20, 2017; Accepted January 9, 2018

DOI: $10.3892 /$ ijmm.2018.3452

\begin{abstract}
MicroRNAs (miRNAs) are considered 'micromanagers of gene expression' and awareness of their fundamental role in the control of biological functions is constantly increasing. Bone formation and homeostasis are complex processes involving the differentiation and interaction of various cell types. Several miRNAs have been shown to be involved in different pathways and stages in the regulation of normal and abnormal bone formation and turnover. This present review focuses on the involvement of miRNAs in terms of their effect on the commitment of bone marrow mesenchymal stem cells towards osteogenesis, adipogenesis and chondrogenesis, respectively. The miRNAs involved in regulating osteoblast, chondroblast and osteoclast activity, are also taken into consideration, as are their interactions. miRNA expression levels, which may differ significantly in healthy versus pathological conditions, can be readily monitored and represent useful biomarkers. Several studies have suggested that miRNAs offer potential as useful biomarkers of bone pathologies, including osteoporosis and osteosarcoma. The development of efficient methods of delivering miRNA mimics or miRNA inhibitors into specific cells remains a challenge for novel therapeutic applications in the field of personalized medicine.
\end{abstract}

\section{Contents}

1. Introduction

2. Role of miRNAs in commitment determination of MSCs

3. miRNAs which promote adipogenesis and inhibit osteogenesis

4. miRNAs which promote osteogenesis and inhibit adipogenesis

Correspondence to: Professor Monica Mottes, Department of Neurosciences, Biomedicine and Movement Sciences, Biology and Genetics Section, University of Verona, 8 Strada Le Grazie, Verona I-37134, Italy

E-mail: monica.mottes@univr.it

Key words: microRNAs, mesenchymal stem cell, osteoblast, chondrocyte, osteoclast, osteoporosis
5. miRNAs which regulate osteoblast activity

6. miRNAs which regulate chondrocyte activity

7. miRNAs which regulate osteoclast activity

8. Role of miRNAs in the pathological osteogenic commitment of progenitor cells

9. Altered miRNA expression levels in osteoporosis

10. miRNAs as potential therapeutic targets for osteoporosis 11. Conclusions

\section{Introduction}

Skeletal development is a multistep process during which mesenchymal progenitor cells (MSCs) undergo proliferation and differentiation, giving rise to cartilage and bone cells. Bone is produced by two distinct processes. In the endochondral ossification process, which occurs in long bones and vertebrae, MSC-derived chondrocytes produce a cartilage template, which is subsequently replaced by a mineralized matrix, deposed by the bone-making cells, MSC-derived osteoblasts. The intramembranous ossification process, which occurs in skull bones and clavicle formation, relies instead on the direct differentiation of condensed MSCs into osteoblasts. The bone modeling occurring during development and the life-long process of remodeling are controlled by several factors, including systemic hormones, bone morphogenetic proteins (BMPs), fibroblast growth factors (FGFs) and secreted signaling factors, including Wnt. Various signaling molecules can trigger intracellular responses by modulating the expression of transcription factors, which are essential for MSC chondrogenic/osteogenic commitment; these include Runt-related transcription factor 2 (RUNX2), SRY-box 9 (SOX9), osterix (OSX) and activating transcription factor 4 (ATF4). Bone plasticity allows continuous adjustments to the mechanical demands solicited by skeletal functions. The leading effectors of bone remodeling are osteoblasts, osteocytes (mature osteoblasts within the mineralized matrix) and osteoclasts (large multinucleated cells suited to bone resorption due to their ability to secrete hydrochloric acid and proteases which degrade the mineralized matrix). Interactions occurring between these three cell types contribute to their reciprocal regulation and determine bone homeostasis in physiological conditions. Osteoclast differentiation is induced by the osteoblastic product, receptor activator of nuclear factor- $\mathrm{\kappa B}$ (RANK) 
ligand (RANKL), which interacts with its osteoclastic receptor, RANK. Osteoblasts can regulate osteoclast differentiation by producing osteoprotegerin, a decoy receptor for RANKL, thereby preventing its interaction with RANK. Osteocytes, far from being inactive cells trapped in mineralized tissue, control osteoblast and osteoclast activity, and they are critical in the regulation of the Wnt signaling pathway. Sclerostin (SOST), a Wnt negative regulator, is expressed specifically by mature osteocytes (Fig. 1). Mechanical forces and anabolic agents inhibit the production of SOST. Disturbances in these complex interactions may cause various bone diseases, including osteoporosis, osteopetrosis, osteogenesis imperfecta and Paget's disease (1-3). Epigenetic factors, including microRNAs (miRNAs) are also essential in bone formation and homeostasis (4). These are small, non-coding RNAs, which act as negative regulators of the expression of specific target mRNAs. A single miRNA can act as a post-transcriptional repressor by binding to partially complementary sequences in the 3'UTR sites of various mRNAs. miRNAS are involved in MSC commitment, osteoblast and osteocyte activity, osteoclastic maturation and bone cell interactions with their microenvironment (3-6). Altered patterns in the expression of miRNAs have been found in in vivo and in vitro models of bone disorders (7). There is increasing interest in the promising potential applications of miRNAs as diagnostic biomarkers and as molecular targets for therapeutic options in the treatment of bone disorders. miRNAs can be isolated from cells or bone specimens; they can also be retrieved from circulatory biofluids, including plasma or serum, as they are secreted from cells in exosomes or encapsulated within microvesicles. The present review examines and discusses the role of selected miRNAs, which have been consistently recognized in previous studies to affect the activity of various bone cell types in physiological and pathological conditions (7-9).

\section{Role of miRNAs in commitment determination of MSCs}

Bone cells progenitors, MSCs, are multipotent stem cells capable of differentiating into adipocytes, chondrocytes or osteoblasts. A mutually inhibitory association exists between osteogenic and adipogenic commitment. Alternative cell fate decisions are regulated by multiple signaling pathways. Increasing evidence indicates that miRNAs affect decisions concerning the fate of bone marrow MSCs. These can operate by silencing components of the Wnt or BMP signaling pathways at the post-transcriptional level, and by modulating the expression of key transcription factors, including RUNX2 (10-12). Chondrocytes arise from mesenchymal cell aggregation and differentiation. Growth factors, cellular interactions and extracellular matrix (ECM) elements are involved in the differentiation process by inducing chondrocyte-specific gene expression of SOX9, COL2A1, aggrecan, COL10A1 and parathyroid hormone-related protein (13). In this scenario, miRNAs are important in chondrocyte differentiation. In particular, miR-30a has been shown to be significantly upregulated during the chondrogenic differentiation of rat MSCs; it targets delta-like 4 gene, a ligand of the Notch signaling family (14). miR-140, which targets A disintegrin and metalloproteinase with thrombospondin motif 5, a metalloproteinase which degrades aggrecan, is expressed at a high level during the chondrogenesis of MSCs, in addition to increased expression levels of SOX9 and COL2A1 (15). By contrast, miR-455-3p has been shown function as an early activator of chondrogenesis, by targeting the gene expression of RUNX2 (16). The miRNAs involved in the adipogenic osteogenic and chondrogenic switch, respectively, in addition to their targets, are shown in Fig. 2.

\section{3. miRNAs which promote adipogenesis and inhibit osteogenesis}

miR-204 and miR-211 behave as endogenous repressors of RUNX2, the master regulator of MSC osteogenic commitment (12). miR-17-p5 and miR-106-a target BMP2 mRNA, which promotes osteogenic differentiation (17). miR-30e targets LDL receptor-related protein 6 (LRP6), a member of the Wnt canonical pathway co-receptor group (LRP5/LRP6/Frizzled) (18). miR-637 is known to target OSX, a key regulator of osteoblastic maturation acting downstream of RUNX2 (19).

\section{4. miRNAs which promote osteogenesis and inhibit adipogenesis}

miR-21 targets SRY-box 2 (SOX2), one of the four genes which promote induced pluripotent stem cells, and sprouty homolog 2 (SPRY2), a negative regulator of the extracellular signal-regulated kinase-mitogen-activated protein kinase signaling pathway $(20,21)$. In MSCs overexpressing miR-21, osteogenic markers, including RUNX2 and osteonectin (OCN), are also overexpressed (22). This suggests a dual action of miR21 on progenitor cells, namely the suppression of pluripotency and promotion of osteogenic differentiation. miR-22 is also present in bone marrow progenitors; its expression decreases during adipogenic differentiation and increases during osteogenic differentiation (23). miR-22 and miR-2861 target histone deacetilase 6 (HDAC6) and histone deacetilase 5 (HDAC5), respectively. These histone deacetylases act as transcriptional co-repressors of RUNX2 (24). miR-3960, which is clustered with miR-2861 and encoded by the same transcript, targets homeobox A2 gene, another RUNX2 inhibitor (25). miR-20a promotes osteogenic differentiation by targeting peroxisome proliferator activated receptor $\gamma 2$, a positive regulator of adipocyte differentiation (26).

\section{5. miRNAs which regulate osteoblast activity}

The miRNAs involved in regulating osteoblast activity are listed in Table I. OsteomiRNAs, or bone-regulating miRNAs, can act as stimulators and repressors of osteogenesis. Among the stimulators, miR322 appears to increase the expression of OSX by targeting transducer of ERBB2, 2 (TOB2), which belongs to the TOB family of antiproliferative proteins; it facilitates the deadenylation and degradation of mRNAs, including OSX (27). By contrast, miR-181 promotes osteogenesis by targeting mRNAs coding for members of the transforming growth factor- $\beta$ (TGF- $\beta$ ) signaling pathway, namely TGF- $\beta$ receptor 1 and TGF- $\beta$-induced, which negatively regulate osteoblastogenesis (28). miR-29 exerts a positive regulatory effect on osteoblast differentiation, enhancing Wnt signaling, 


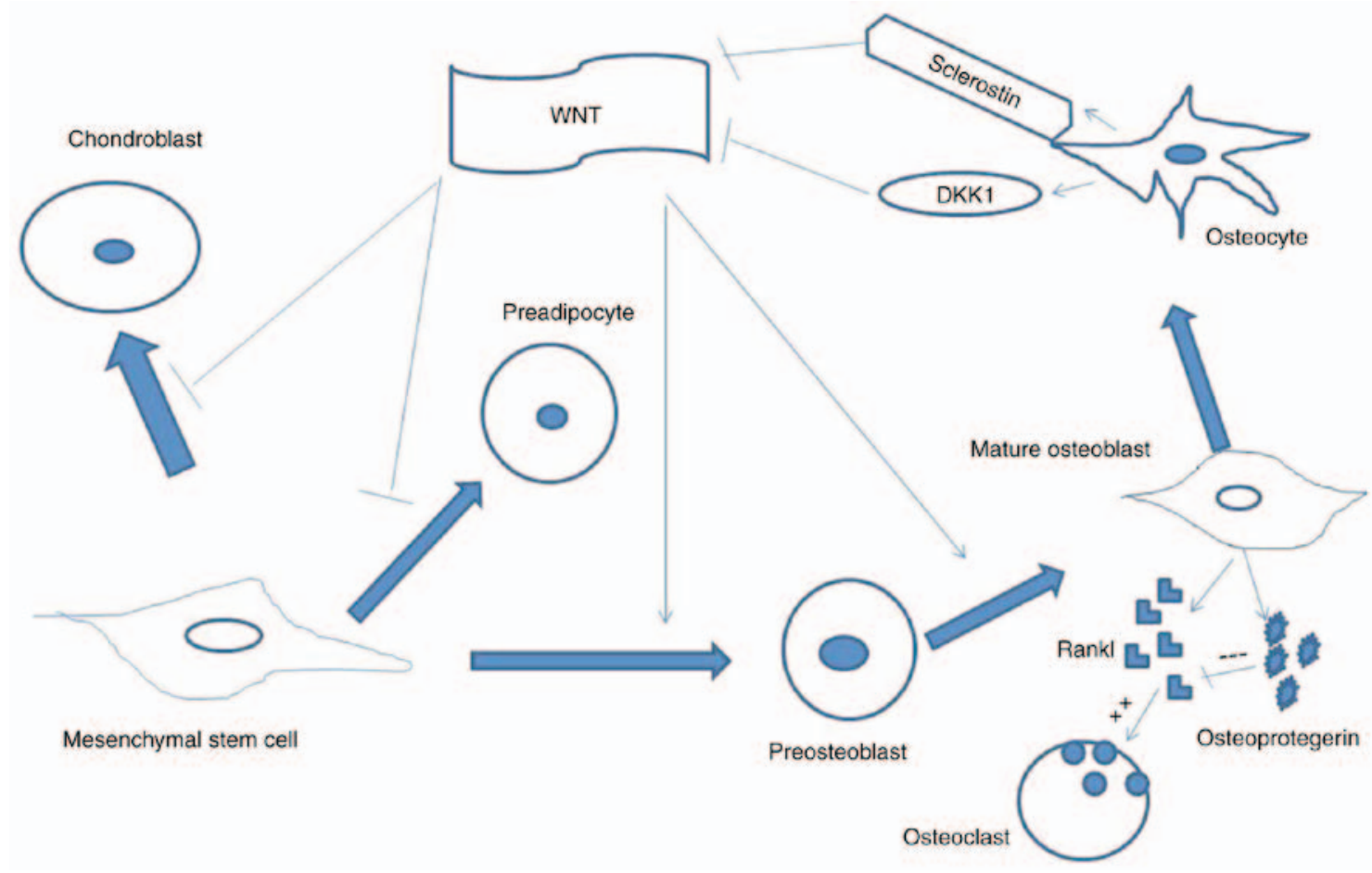

Figure 1. Schematic representation of Wnt signaling pathway control in mesenchymal stem cell commitment and bone homeostasis. DKK1, Dickkopf-related protein 1; Rank1, receptor activator of nuclear factor- $\varkappa \mathrm{B}$ ligand.

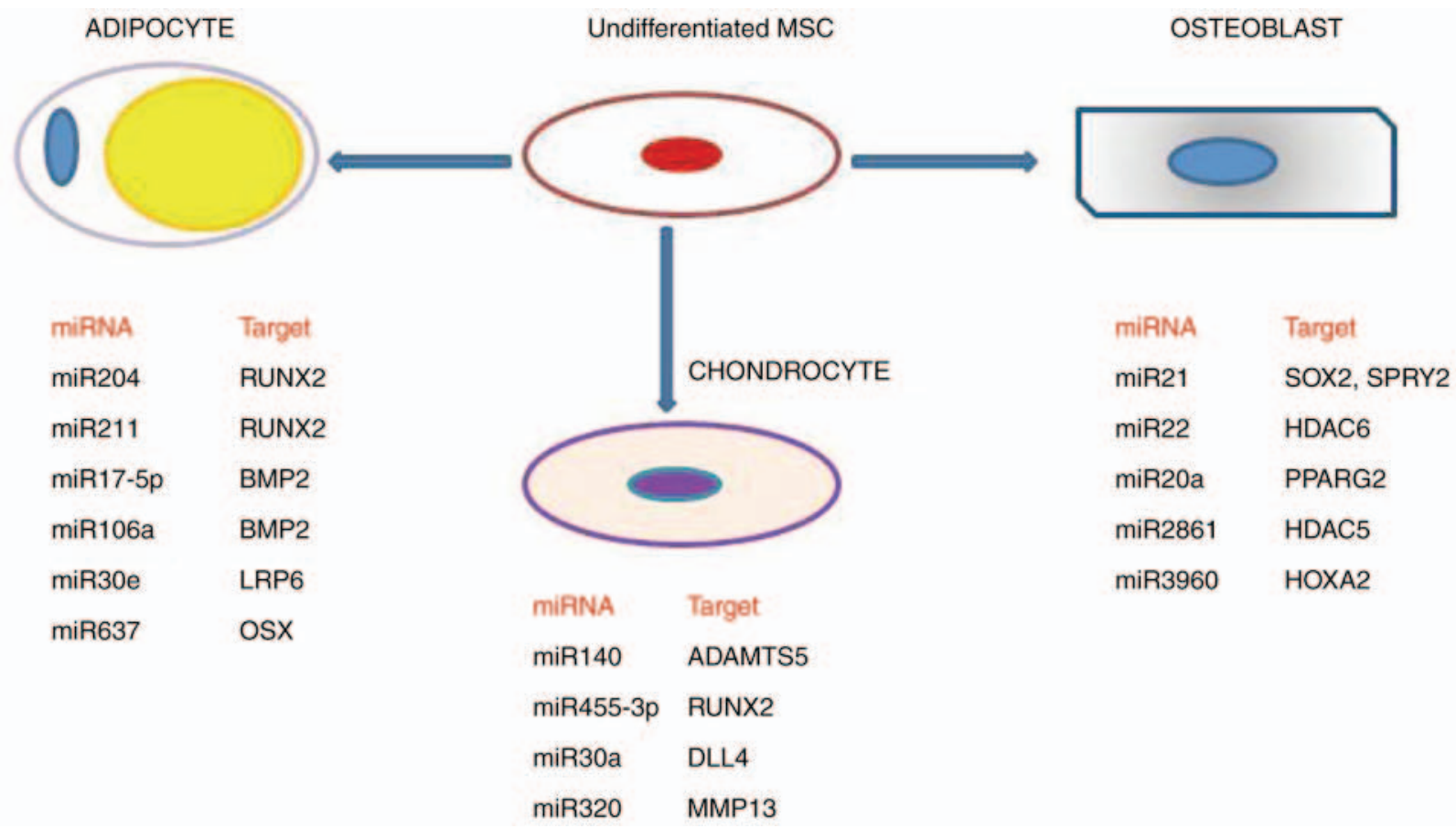

Figure 2. Role of different miRNAs in contribution to the commitment determination of undifferentiated MCSc towards adipogenesis, chondrogenesis and osteogenesis, respectively. MSC, mesenchymal stem cell; miR, microRNA; RUNX2, Runt-related transcription factor 2; BMP2, bone morphogenetic protein 2; LRP6, LDL receptor-related protein 6; OSX, osterix; ADAMTS5, A disintegrin and metalloproteinase with thrombospondin motif 5; DLL4, delta-like 4; MMP13, matrix metalloproteinase 13; SOX2, SRY-box 2; SPRY2, sprouty homolog 2; HDAC6, histone deacetilase 6; PPARG2, peroxisome proliferator activated receptor $\gamma 2$; HDAC5, histone deacetilase; HOXA2, homeobox A2.

and Dickkopf-related protein 1 (DKK1), a Wnt inhibitor, is among its targets (10). miR-335-5p also targets DKK1 (29). miR-26-a promotes osteogenesis by targeting glycogen synthase kinase $3 \beta$, a member of the $\beta$-catenin destruction complex (30). The miRNAs which target RUNX2 and OSX, respectively, act as negative regulators of osteogenesis; among others, these include the above-mentioned miR-204 and miR-211, which downregulate RUNX2, whereas miR-138 
Table I. miRNAs which regulate osteoblast activity.

\begin{tabular}{llc}
\hline miRNA & Target gene & $\begin{array}{c}\text { Regulatory effect } \\
\text { on osteogenesis }\end{array}$ \\
\hline miR-322 & TOB2 & $\uparrow$ \\
miR-181 & TGF $\beta R$ & $\uparrow$ \\
TGF $\beta$ I & \\
miR-29 & DKK1 & $\uparrow$ \\
miR-26a & & \\
miR-320-a & GSK3B & $\uparrow$ \\
miR-138 & RUNX2 & $\downarrow$ \\
miR-143 & OSX & $\downarrow$ \\
miR-483-5p & IGF2 & $\downarrow$ \\
miR-182 & FOXO1 & $\downarrow$ \\
miR-155 & SMAD5 & $\downarrow$ \\
\end{tabular}

Examples of miRNAs which modulate osteoblast activity. $\uparrow$, positive effect on osteoblastogenesis; $\downarrow$, negative effect on osteoblastogenesis. miRNA, microRNA; TOB2, targeting transducer of ERBB2, 2; TGF $\beta R$, transforming growth factor $\beta$ receptor; DKK1, Dickkopf-related protein 1; GSK3B, glycogen synthase kinase $3 \beta$; OSX, osterix; IGF2, insulin-like growth factor 2; FOXO1, Forkhead box protein O1; SMAD5, small mothers against decapentaplegic 5 .

and miR-143 target OSX $(31,32)$. A negative regulator of bone formation is miR-214, which targets ATF4, a transcription factor modulating the gene expression of osteocalcin (33). miR-483-5p and miR-320-a downregulate insulin-like growth factor 2 (IGF2) and RUNX2, respectively, which inhibits osteoblastic function (34). Finally, miR-182 negatively regulates osteoblast proliferation by targeting Forkhead box protein O1 (FOXO1), a regulator of bone mass (35); miR-155 has been demonstrated to inhibit mouse osteoblast differentiation by suppressing the expression of small mothers against decapentaplegic family member 5 (SMAD5) (36).

\section{6. miRNAs which regulate chondrocyte activity}

The miRNAs involved in regulating chondrocyte activity are listed in Table II. The differentiation process in chondrocytes is followed by proliferation, hypertrophy, terminal differentiation, mineralization and programmed cell death. Of note, miR-140 (mentioned above) is involved in cartilage homeostasis; it has been shown that miR-140-knockout mice developed osteoarthritis (37). miR-let7, expressed in various tissues, is required for chondrocytes proliferation in normal conditions (38). The downregulation of miR-let7, obtained by Lin28a inhibitor, reduces chondrocytes proliferation and growth. These effects may be interpreted as consequences of the overexpression of two miR-let 7 target genes, namely cell division cycle 34 and E2F transcription factor 5. Therefore, miR-let7, together with miR140, regulates the skeletal development by acting on chondrocyte proliferation and homeostasis (38). miR-199a and miR-214, which are generated from the RNA transcript Dnm3os and expressed in mesenchymal cells and chondrocytes, contribute to correct
Table II. miRNAs which regulate chondrocyte activity.

\begin{tabular}{llc}
\hline miRNA & \multicolumn{1}{c}{ Target gene } & $\begin{array}{c}\text { Regulatory effect } \\
\text { on chondrogenesis }\end{array}$ \\
\hline miR-140 & ADAMTS-5 & $\uparrow$ \\
miR-1et7 & CDC34 & $\uparrow$ \\
& E2F5 & $\uparrow$ \\
miR-199a & SMAD1, COX2 & \\
miR-214 & OSX, ATF4 & $\downarrow$ \\
miR-195 & GIT1 & $\downarrow$ \\
miR-138 & HIF-1 $\alpha$ & \\
& SP1 & $\downarrow$ \\
miR-34a & HIF-2 $\alpha$ & \\
\hline
\end{tabular}

Examples of miRNAs which modulate chondrocyte activity. $\uparrow$, positive effect on chondrogenesis; $\downarrow$, negative effect on chondrogenesis. miR, microRNA; ADAMTS-5, A disintegrin and metalloproteinase with thrombospondin motif 5; CDC34, cell division cycle 34; E2F5, E2F transcription factor 5; SMAD1, small mothers against decapentaplegic 1; COX2, cyclooxygenase 2; OSX, osterix; ATF4, activating transcription factor 4; GIT1, G-protein coupled receptor kinase interacting protein-1; HIF, hypoxia inducible factor; SP1, specificity protein 1; SIRT1, sirtuin 1.

skeletal development. In particular, the upregulation of miR-199 has been observed upon chondrocytic differentiation (39). miR-195 can inhibit chondrocyte proliferation, by acting on the G-protein coupled receptor kinase interacting protein-1 (GIT1) (40) and promote apoptosis by targeting hypoxia inducible factor $1 \alpha$ (HIF-1 $\alpha)$ (41). miR-138 has been shown to act as a negative modulator of the chondrocytic phenotype, as it targets specificity protein 1 and hypoxia-inducible factor $2 \alpha$ (HIF-2 $\alpha$ ), two transcription factors necessary for COL2A1 gene expression. The overexpression of miR-138 has been shown to coincide with loss of the differentiated phenotype of cultured human articular chondrocytes (42). Proliferation and apoptosis are important processes in chondrocyte homeostasis. It has been demonstrated that miR-34a reduces the proliferation rate and induces the apoptosis of primary human chondrocytes by regulating the sirtuin 1/p53 signaling pathway (43).

\section{7. miRNAs which regulate osteoclast activity}

An important function in bone homeostasis is bone resorption. Various evidence supports the idea that miRNAs are involved in bone resorption, as they may act on osteoclastic proliferation, differentiation and survival (Table III). Among the positive regulators of osteoclastogenesis is miR-223, which targets nuclear factor IA (NFIA), a negative regulator of macrophage colony-stimulating factor receptor (44). miR-148-a has been demonstrated to stimulate osteoclastogenesis by targeting transcription factor MafB, a transcriptional repressor of RANKL (45). miR-31 is upregulated during osteoclast differentiation in murine bone marrow cells; its target, RhoA, is a GTPase involved in cytoskeletal reorganization, which can affect osteoclast formation (46). Similarly, miR-21 is 
Table III. miRNAs which regulate osteoclast activity.

\begin{tabular}{llc}
\hline miRNA & Target gene & $\begin{array}{c}\text { Regulatory effect } \\
\text { on osteogenesis }\end{array}$ \\
\hline miR-223 & NF1A & $\uparrow$ \\
miR-148a & MAFB & $\uparrow$ \\
miR-31 & RhoA & $\uparrow$ \\
miR-21-5p & PDCD4 & $\uparrow$ \\
miR-503 & RANK & $\downarrow$ \\
miR-218 & NFATC1 & $\downarrow$ \\
miR-124-3p & & $\downarrow$ \\
\hline
\end{tabular}

Examples of miRNAs which modulate osteoclast activity. $\uparrow$, positive effect on osteoclastogenesis; $\downarrow$, negative effect on osteoclastogenesis . miR, microRNA; NF1A, nuclear factor 1A; PDCD4, programmed cell death 4; RANK, receptor activator of nuclear factor- $\mathrm{kB}$; NFATC1, the nuclear factor of activated T-cells 1; TRAF6, TNF receptor-associated factor 6 .

upregulated in osteoclast precursors and is identified as a marker of RANKL-induced osteoclastogenesis, targeting the programmed cell death 4 (PDCD4) gene (47). By contrast, miR-503 is a negative regulator of osteoclastogenesis, as it targets the osteoclastic receptor RANK (48). miR-218 and miR-125-a also act as negative regulators; miR-218 targets the nuclear factor of activated T-cells 1 (NFATC1) signaling molecule, which is required for osteoclastogenesis, and regulates several genes involved in osteoclast differentiation and function (49). miR-125-a targets TNF receptor-associated factor 6, an osteoclastogenesis-promoting factor (50).

\section{Role of miRNAs in the pathological osteogenic commitment of progenitor cells}

Altered patterns of miRNA expression have been shown in bone-related pathologies, which may jeopardize progenitor cell commitment and osteogenic differentiation. miR-29b is known to have an important regulatory role in osteoblast differentiation, as it targets COL1A1 and SPARC (osteonectin) mRNAs (51). As osteoblasts mature, miR-29b attenuates the expression of collagen genes, allowing the organization of collagen fibrils for subsequent mineralization. Of note, the downregulation of miR-29b has been reported in two distinct genetic bone disorders, osteogenesis imperfecta (OI) and osteopetrosis (OPT). OI is an 'osteoblast disease', characterized by varying degrees of bone fragility and skeletal deformities. Kaneto et al (52) reported that, in patients with OI, heterozygosity for causative COL1A1 gene mutations led to the reduced mRNA expression of COL1A1, and the levels of miR-29b were severely reduced. The authors suggested that the miR-29b control of collagen protein accumulation in the ECM depends on the levels of its target and hypothesized that the low mRNA levels of COL1A1 observed in OI cells at various stages of osteogenic differentiation were not sufficient for the induction of miR-29b. OPT is considered an 'osteoclast disease'. In peripheral blood mononuclear cells (PBMCs) from patients with OPTA2, a survey of aberrantly expressed miRNAs showed that miR-29b was among the most significantly downregulated (53). A previous study demonstrated that miR-29b promotes osteoclastogenesis; its knockdown in murine pre-osteoclasts inhibited differentiation (54). Due to their important effects on bone marrow progenitor cell differentiation, misregulated miRNAs may profoundly affect primary and metastatic bone tumors. It has been demonstrated that the upregulation of miR-135b is involved in the impaired osteogenic differentiation of MSCs derived from patients with multiple myeloma (55). The study also demonstrated that impaired osteogenic differentiation overlapped with a marked downregulation of osteogenic markers, including BSP, COL1A1 and OPN. The authors suggested that miR-135b inhibited osteogenic differentiation by targeting SMAD5 mRNA and possibly other strategic target mRNAs. Bone metastasis, frequently occurring in late stages of breast and prostate cancer, disrupts normal bone remodeling. The involvement of miRNAs in the control and fate of bone metastasis has been reviewed extensively $(56,57)$. miRNA profiles can be of diagnostic and prognostic value, and they may become therapeutic agents in the near future.

Osteosarcoma (OS) is a common malignant bone tumor in children and young adults. Surgery and chemotherapy failure occur in certain patients with OS. Therefore, biomarkers for active disease are required in order to monitor relapses and to predict prognosis in subjects who have a poor response to multi-agent chemotherapy. Several studies have investigated the role of circulating miRNAs as possible biomarkers with various, sometimes contradictory, findings (58-61). In particular, miR21 (Fig. 2) emerges consistently as a significant marker for poor prognosis, when its expression levels in the plasma of patients with OS and cancer cells are compared with those found in controls (62-64). miR-199a, which has been shown to be dysregulated in several types of tumor, has been found to be underexpressed in osteosarcoma cells and patient samples. Keremu et al (65) demonstrated that cisplatin-resistant OS cells treated with agomiR199 were sensitized to cisplatin. Further investigations in this field may lead to the identification of other miRNA markers for more precise and timely prognosis, and to miRNAs therapeutic applications for osteosarcoma.

\section{Altered miRNA expression levels in osteoporosis}

Osteoporosis is a common age-related degenerative disease associated with bone loss and low-traumatic fractures. Bone fragility in postmenopausal osteoporosis originates from an imbalance in bone homeostasis, caused by increased osteoclastic activity and a progressive decline in osteoblastic proliferation (66). Due to the importance of miRNAs in the regulation of bone remodeling, several studies have investigated osteoporosis-related changes in their expression (Table IV). In several studies specific miRNAs, selected on the basis of previous reports, were isolated from serum samples of osteoporotic patients (OP) and age/sex matched healthy controls, reverse transcribed and then analyzed using reverse transcription-quantitative polymerase chain reaction analysis (67-72). In another study, total RNA was extracted from fresh femoral trabecular bone obtained from OP and 
Table IV. miRNAs as biomarkers of osteoporosis.

\begin{tabular}{llll}
\hline Cell type & Target gene & $\begin{array}{c}\text { miRNAs differentially expressed in patients } \\
\text { with osteoporosis }\end{array}$ & $\begin{array}{c}\text { Expression levels } \\
\text { (compared with controls) }\end{array}$ \\
\hline Osteoblast & FOXO1 & $\begin{array}{l}\text { miR-483-5p } \\
\text { miR-320 a }\end{array}$ & $\uparrow$ \\
& CTNNB1 & miR-30e & $\uparrow$ \\
& LRP6 & miR-2861 & $\uparrow$ \\
Osteoclast & SPRY, PDCD4 & miR-21-5p & miR-335-5p \\
& DKK1 & miR-148a & $\uparrow$ \\
& NFATC1 & miR-124-3p & $\uparrow$ \\
\hline
\end{tabular}

Examples of miRNAs differentially expressed in patients with osteoporosis. $\uparrow$, increased expression; $\downarrow$, decreased expression; miR, microRNA; FOXO1, Forkhead box protein O1; CTNNB1, LRP6, LDL receptor-related protein 6; HADC5; SPRY, sprouty homolog 2; PDCD4, programmed cell death 4; DKK1, Dickkopf-related protein 1; NFATC1, nuclear factor of activated T-cells 1. Increased levels of miR2861 and 124-3p are discussed in section 9 .

control groups, and hybridized to a miRNA array containing $>1,900$ miRNAs (34). Biostatistical analyses were performed in all experiments in order to identify significant $(\mathrm{P}<0.05)$ differences in miRNA expression between cases and controls. Several limitations in these studies do not allow the obtaining of univocal miRNAs signatures of osteoporosis. Notably, the patient/control groups in each study were small and different; in addition, miRNAs were different and non-randomly selected. However, consistent findings stand out from the plethora of data. Significant differences in the expression of specific miRNAs produced either by osteoblast or osteoclast cells, as described above, and in Tables I and III, respectively, appear to be essential in osteoporosis. Table IV illustrates how osteoblastic miRNAs, which target essential positive effectors of osteogenic commitment, including miR30e, miR214, miR483-5p, miR182 and miR320a, are overexpressed in patients with osteoporosis, compared with matched healthy controls, whereas other miRNAs, including miR335-5p, which targets DKK1, are underexpressed in OP. Certain findings conflict with previously reported data. Yavropoulou et al (72) found serum levels of miR124-3p, a negative regulator of osteoclastogenesis (Table III) and miR2861, a positive regulator of osteoblastogenesis (Fig. 2) to be higher in postmenopausal women with a low bone mineral density (BMD), compared with those in women with a normal BMD. The authors hypothesized a compensatory mechanism of enhanced osteogenesis in response to menopause-induced bone loss, in order to justify their conflicting findings.

\section{0. miRNAs as potential therapeutic targets for osteoporosis}

As the dysregulation of miRNAs appears to contribute considerably to bone pathologies, including osteoporosis, several approaches aiming to correct such dysregulation have been applied to model systems. One type of approach consists in the delivery of pro-osteoblastic miRNAs in order to promote osteogenesis; another is the silencing of endogenous pro-osteoclastic miRNAs. The design of biologically
sTable RNA molecules and their efficient delivery represent the main requirements in the two approaches. Zhang et al (73) designed a two-stage delivery system. A hyperbranched polymer vector containing miRNA26-a was encapsulated in polylactic-co-glycolic acid (PLGA) microspheres; these biodegradable PLGA microspheres were attached to 3D scaffolds, which were then implanted into mice. miRNA26-a had previously been demonstrated to promote osteogenesis (Table I). Zhang et al (73) showed that long-term miR26-a delivery locally rescued the osteogenic capacity in osteoporotic mice. By contrast, Liu et al (74) described a successful strategy to knock down pro-osteoclastic miRNA148-a (Table III). Downregulation/silencing of single miRNAs can be achieved by means of antagomiRs, specifically engineered oligonucleotides complementary to the miRNA target. Liu et al (74) encapsulated antagomiR-148 a in (D-Asp $)_{8}$-modified liposomes, which favorably bind to bone-resorption surfaces, thus targeting osteoclasts. The therapeutic effect of antagomiR-148a was assessed upon delivery to ovariectomized (OVX) mice, which are animal models for the postmenopausal status. Encouraging results were derived from bone metabolism marker measurements and the observed attenuated decrease of BMD in the treatment group. In another study, Chen et al (48) demonstrated an opposite effect of antagomiR503 upon injection in OVX mice. miRNA503 inhibits osteoclastogenesis by targeting RANK, as described above (Table III) and the authors found it was dramatically downregulated in postmenopausal osteoporotic subjects. AntagomiR503-treated animals showed increased protein expression of RANK and bone resorption, whereas treatment with exogenous agomiR503 inhibited bone resorption and prevented bone loss. The above cited studies suggest that certain miRNAs are important in the pathogenesis of postmenopausal osteoporosis, due to their various effects on osteoblast or osteoclast activities. Exogenous agomiRNAs (potentiators), or exogenous antagomiRNAs (inhibitors) appear to be novel therapeutic tools against osteoporosis, a disabling disease which affects 200 million individuals worldwide (75). 


\section{Conclusions}

The role of miRNAs as modulators of gene expression and, consequently of physiological and pathological tissue functions, is attracting increasing attention. Referred to as 'micromanagers of gene expression', miRNAs regulate a substantial region of the human genome. Experimental in vivo and in vitro studies have shown how miRNAs complex interactions affect bone development and homeostasis. Excreted miRNAs, recovered from blood or other body fluids, represent useful biomarkers for skeletal disorders; experiments in animal models suggest that antagomiRNAs or miRNA mimics may function as novel therapeutic tools. The exploitation of miRNA analysis as a molecular diagnostic tool is an interesting concept; however, important issues require consideration, including sample-to-sample biological variability and modulation of miRNAs in similar phenotypes. Although certain miRNAs have been observed consistently in different experimental conditions, their stimulatory or inhibitory effects on osteoblast and osteoclast differentiation remain to be fully elucidated. For example, miR-335-5p is upregulated in the commitment of MSCs to the osteogenic lineage, however, its levels are reduced during osteoblast maturation (76). As another example, miR-223 is expressed in mononuclear osteoclast precursors and has been shown to enhance osteoclast differentiation (77). By contrast, in PBMCs or RAW264.7 cells, the overexpression of miR-223 inhibits osteoclast formation (6). Therefore, these findings define the role of miRNAs in maintaining bone homeostasis by modulating osteoblast and osteoclast commitment or maturation; however, complementary evaluations are advisable in order to interpret correctly those findings concerning the elevation or reduction of specific miRNAs. Finally, an awareness of the roles and functions of miRNAs is a pre-requisite for the development of promising tools in personalized medicine. A number of issues remain, including how to optimize miRNA stability and delivery systems efficiency, and how to reach a specific tissue, specific cell population and specific mRNA target. As individual miRNAs may target numerous mRNAs, adverse collateral effects require consideration. These challenges indicate the requirement for further basic investigations in the field.

\section{Acknowledgements}

This review was supported by Fondo Unico della Ricerca grants of the University of Verona (Verona, Italy) to Professor L.D.C. and Professor M.M., respectively.

\section{Competing interests}

The authors declare that they have no competing interests.

\section{References}

1. Valenti MT, Dalle Carbonare L and Mottes M: Osteogenic differentiation in healthy and pathological conditions. Int J Mol Sci 18: E41, 2016.

2. Idolazzi L, Fassio A, Tripi G, Braga V, Viapiana O, Adami G, Rossini $\mathrm{M}$ and Gatti D: Circulating Dickkopf-1 and sclerostin in patients with Paget's disease of bone. Clin Rheumatol 36: 925-928, 2017
3. Mäkitie RE, Haanpää M, Valta H, Pekkinen M, Laine CM, Lehesjoki AE, Schalin-Jäntti C and Mäkitie O: Skeletal characteristics of WNT1 osteoporosis in children and young adults. J Bone Miner Res 31: 1734-1742, 2016.

4. Papaioannou G, Mirzamohammadi F and Kobayashi T: MicroRNAs involved in bone formation. Cell Mol Life Sci 71: 4747-4761, 2014.

5. Yuan Y, Zhang L, Tong X, Zhang M, Zhao Y, Guo J, Lei L, Chen X, Tickner J, Xu J and Zou J: Mechanical stress regulates bone metabolism through MicroRNAs. J Cell Physiol 232: 1239-1245, 2017.

6. Ji X, Chen X and Yu X: MicroRNAs in osteoclastogenesis and function: Potential therapeutic targets for osteoporosis. Int J Mol Sci 17: 349, 2016.

7. Gennari L, Bianciardi S and Merlotti D: MicroRNAs in bone diseases. Osteoporos Int 28: 1191-1213, 2017.

8. Wu C, Tian B, Qu X, Liu F, Tang T, Qin A, Zhu Z and Dai K: MicroRNAs play a role in chondrogenesis and osteoarthritis (Review). Int J Mol Med 34: 13-23, 2014.

9. Huang C, Geng J and Jiang S: MicroRNAs in regulation of osteogenic differentiation of mesenchymal stem cells. Cell Tissue Res 368: 229-238, 2017.

10. Kapinas K, Kessler C, Ricks T, Gronowicz G and Delany AM: miR-29 modulates Wnt signaling in human osteoblasts through a positive feedback loop. J Biol Chem 285: 25221-25231, 2010.

11. Gong Y, Xu F, Zhang L, Qian Y, Chen J, Huang H and Yu Y: MicroRNA expression signature for Satb2-induced osteogenic differentiation in bone marrow stromal cells. Mol Cell Biochem 387: 227-239, 2014.

12. Huang J, Zhao L, Xing L and Chen D: MicroRNA-204 regulates Runx 2 protein expression and mesenchymal progenitor cell differentiation. Stem Cells 28: 357-364, 2010.

13. Onyekwelu I, Goldring MB and Hidaka C: Chondrogenesis, joint formation, and articular cartilage regeneration. J Cell Biochem 107: 383-392, 2009.

14. Tian Y, Guo R, Shi B, Chen L, Yang L and Fu Q: MicroRNA-30a promotes chondrogenic differentiation of mesenchymal stem cells through inhibiting Delta-like 4 expression. Life Sci 148: 220-228, 2016.

15. Miyaki S, Nakasa T, Otsuki S, Grogan SP, Higashiyama R, Inoue A, Kato Y, Sato T, Lotz MK and Asahara H: MicroRNA-140 is expressed in differentiated human articular chondrocytes and modulates interleukin-1 responses. Arthritis Rheum 60: 2723-2730, 2009

16. Zhang Z, Hou C, Meng F, Zhao X, Zhang Z, Huang G, Chen W, Fu M and Liao W: MiR-455-3p regulates early chondrogenic differentiation via inhibiting Runx2. FEBS Lett 589: 3671-3678, 2015.

17. Li H, Li T, Wang S, Wei J, Fan J, Li J, Han Q, Liao L, Shao C and Zhao RC: miR-17-5p and miR-106a are involved in the balance between osteogenic and adipogenic differentiation of adipose-derived mesenchymal stem cells. Stem Cell Res 10: 313-324, 2013

18. Wang J, Guan X, Guo F, Zhou J, Chang A, Sun B, Cai Y, Ma Z, Dai C, Li X and Wang B: miR-30e reciprocally regulates the differentiation of adipocytes and osteoblasts by directly targeting low-density lipoprotein receptor-related protein 6. Cell Death Dis 10: e845, 2013.

19. Zhang JF, Fu WM, He ML, Wang H, Wang WM, Yu SC, Bian XW, Zhou J, Lin MC, Lu G, et al: MiR-637 maintains the balance between adipocytes and osteoblasts by directly targeting Osterix. Mol Biol Cell 22: 3955-3961, 2011.

20. Mei Y, Bian C, Li J, Du Z, Zhou H, Yang Z and Zhao RC: miR-21 modulates the ERK-MAPK signaling pathway by regulating SPRY2 expression during human mesenchymal stem cell differentiation. J Cell Biochem 114: 1374-1384, 2013.

21. Trohatou O, Zagoura D, Bitsika V, Pappa KI, Antsaklis A, Anagnou NP and Roubelakis MG: Sox 2 suppression by miR-21 governs human mesenchymal stem cell properties. Stem Cells Transl Med 3: 54-68, 2014.

22. Yang N, Wang G, Hu C, Shi Y, Liao L, Shi S, Cai Y, Cheng S, Wang X, Liu Y, et al: Tumor necrosis factor alpha suppresses the mesenchymal stem cell osteogenesis promoter miR-21 in estrogen deficiency-induced osteoporosis. J Bone Miner Res 28: 559-573, 2013.

23. Huang S, Wang S, Bian C, Yang Z, Zhou H, Zeng Y, Li H, Han Q and Zhao RC: Upregulation of miR-22 promotes osteogenic differentiation and inhibits adipogenic differentiation of human adipose tissue-derived mesenchymal stem cells by repressing HDAC6 protein expression. Stem Cells Dev 21: 2531-2540, 2012. 
24. Westendorf JJ: Transcriptional co-repressors of Runx2. J Cell Biochem 98: 54-64, 2006.

25. Hu R, Liu W, Li H, Yang L, Chen C, Xia ZY, Guo LJ, Xie H, Zhou HD, Wu XP and Luo XH: A Runx2/miR-3960/miR-2861 regulatory feedback loop during mouse osteoblast differentiation. J Biol Chem 286: 12328-12339, 2011.

26. Zhang JF, Fu WM, He ML, Xie WD, Lv Q, Wan G, Li G, Wang H, Lu G, Hu X, et al: MiRNA-20a promotes osteogenic differentiation of human mesenchymal stem cells by co-regulating BMP signaling. RNA Biol 8: 829-838, 2011.

27. Gámez B, Rodríguez-Carballo E, Bartrons R, Rosa JL and Ventura F: MicroRNA-322 (miR-322) and its target protein Tob2 modulate Osterix (Osx) mRNA stability. J Biol Chem 288: 14264-14275, 2013.

28. Bhushan R, Grünhagen J, Becker J, Robinson PN, Ott CE and Knaus P: miR-181a promotes osteoblastic differentiation through repression of TGF- $\beta$ signaling molecules. Int J Biochem Cell Biol 45: 696-705, 2013.

29. Zheng L, Tu Q, Meng S, Zhang L, Yu L, Song J, Hu Y, Sui L, Zhang J, Dard M, et al: Runx2/DICER/miRNA pathway in regulating osteogenesis. J Cell Physiol 232: 182-191, 2017.

30. Li Y, Fan L, Liu S, Liu W, Zhang H, Zhou T, Wu D, Yang P, Shen L, Chen J and Jin Y: The promotion of bone regeneration through positive regulation of angiogenic-osteogenic coupling using microRNA-26a. Biomaterials 34: 5048-5058, 2013.

31. Eskildsen T, Taipaleenmäki H, Stenvang J, Abdallah BM, Ditzel N, Nossent AY, Bak M, Kauppinen S and Kassem M: MicroRNA-138 regulates osteogenic differentiation of human stromal (mesenchymal) stem cells in vivo. Proc Natl Acad Sci USA 108: 6139-6144, 2011.

32. Li E, Zhang J, Yuan T and Ma B: MiR-143 suppresses osteogenic differentiation by targeting Osterix. Mol Cell Biochem 390: 69-74, 2014.

33. Wang X, Guo B, Li Q, Peng J, Yang Z, Wang A, Li D, Hou Z, Lv K, Kan G, et al: miR-214 targets ATF4 to inhibit bone formation. Nat Med 19: 93-100, 2013.

34. De-Ugarte L, Yoskovitz G, Balcells S, Güerri-Fernández R, Martinez-Diaz S, Mellibovsky L, Urreizti R, Nogués X, Grinberg D, García-Giralt N and Díez-Pérez A: MiRNA profiling of whole trabecular bone: Identification of osteoporosis-related changes in MiRNAs in human hip bones. BMC Med Genomics 8 : $75,2015$.

35. Jin HL, Kim JS, Kim YJ, Kim SJ, Broxmeyer HE and Kim KS: Dynamic expression of specific miRNAs during erythroid differentiation of human embryonic stem cells. Mol Cells 34: 177-183, 2012.

36. Gu Y, Ma L, Song L, Li X, Chen D and Bai X: miR-155 inhibits mouse osteoblast differentiation by suppressing SMAD5 expression. Biomed Res Int 2017: 1893520, 2017.

37. Miyaki S, Sato T, Inoue A, Otsuki S, Ito Y, Yokoyama S, Kato Y, Takemoto F, Nakasa T, Yamashita S, et al: MicroRNA-140 plays dual roles in both cartilage development and homeostasis. Genes Dev 24: 1173-1185, 2010.

38. Papaioannou G, Inloes JB, Nakamura Y, Paltrinieri E and Kobayashi T: let-7 and miR-140 microRNAs coordinately regulate skeletal development. Proc Natl Acad Sci USA 110: E3291-E3300, 2013.

39. Lin EA, Kong L, Bai XH, Luan Y and Liu CJ: miR-199a, a bone morphogenic protein 2-responsive MicroRNA, regulates chondrogenesis via direct targeting to Smad1. J Biol Chem 284 11326-11335, 2009.

40. Gu YL, Rong XX, Wen LT, Zhu GX and Qian MQ: miR-195 inhibits the proliferation and migration of chondrocytes by targeting GIT1. Mol Med Rep 15: 194-200, 2017.

41. Bai R, Zhao AQ, Zhao ZQ, Liu WL and Jian DM: MicroRNA-195 induced apoptosis in hypoxic chondrocytes by targeting hypoxia-inducible factor 1 alpha. Eur Rev Med Pharmacol Sci 19: 545-551, 2015.

42. Seidl CI, Martinez-Sanchez A and Murphy CL: Derepression of MicroRNA-138 contributes to loss of the human articular chondrocyte phenotype. Arthritis Rheumatol 68 : 398-409, 2016

43. Yan S, Wang M, Zhao J, Zhang H, Zhou C, Jin L, Zhang Y, Qiu X, Ma B and Fan Q: MicroRNA-34a affects chondrocyte apoptosis and proliferation by targeting the SIRT1/p53 signaling pathway during the pathogenesis of osteoarthritis. Int J Mol Med 38: 201-209, 2016.
44. Lian JB, Stein GS, van Wijnen AJ, Stein JL, Hassan MQ, Gaur T and Zhang Y: MicroRNA control of bone formation and homeostasis. Nat Rev Endocrinol 8: 212-227, 2012.

45. Cheng P, Chen C, He HB, Hu R, Zhou HD, Xie H, Zhu W, Dai RC, Wu XP, Liao EY and Luo XH: miR-148a regulates osteoclastogenesis by targeting V-maf musculoaponeurotic fibrosarcoma oncogene homolog B. J Bone Miner Res 28: 1180-1190, 2013.

46. Mizoguchi F, Murakami Y, Saito T, Miyasaka N and Kohsaka H: miR-31 controls osteoclast formation and bone resorption by targeting RhoA. Arthritis Res Ther 15: R102, 2013.

47. Sugatani T, Vacher J and Hruska KA: A microRNA expression signature of osteoclastogenesis. Blood 117: 3648-3657, 2011.

48. Chen C, Cheng P, Xie H, Zhou HD, Wu XP, Liao EY and Luo XH: MiR-503 regulates osteoclastogenesis via targeting RANK. J Bone Miner Res 29: 338-347, 2014

49. Qu B, Xia X, Yan M, Gong K, Deng S, Huang G, Ma Z and Pan X: miR-218 is involved in the negative regulation of osteoclastogenesis and bone resorption by partial suppression of p38MAPK-c-Fos-NFATc1 signaling: Potential role for osteopenic diseases. Exp Cell Res 338: 89-96, 2015.

50. Guo LJ, Liao L, Yang L, Li Y and Jiang TJ: MiR-125a TNF receptor-associated factor 6 to inhibit osteoclastogenesis. Exp Cell Res 321: 142-152, 2014.

51. Laxman N, Rubin CJ, Mallmin H, Nilsson O, Pastinen T, Grundberg E and Kindmark A: Global miRNA expression and correlation with mRNA levels in primary human bone cells. RNA 21: 1433-1443, 2015.

52. Kaneto CM, Lima PS, Zanette DL, Prata KL, Pina Neto JM, de Paula FJ and Silva WA Jr: COL1A1 and miR-29b show lower expression levels during osteoblast differentiation of bone marrow stromal cells from Osteogenesis Imperfecta patients. BMC Med Genet 15: 1471-2350, 2014.

53. Ou M, Zhang X, Dai Y, Gao J, Zhu M, Yang X, Li Y, Yang T and Ding M: Identification of potential microRNA-target pairs associated with osteopetrosis by deep sequencing, iTRAQ proteomics and bioinformatics. Eur J Hum Genet 22: 625-632, 2014.

54. Franceschetti T, Kessler CB, Lee SK and Delany AM: miR-29 promotes murine osteoclastogenesis by regulating osteoclast commitment and migration. J Biol Chem 288: 33347-33360, 2013

55. Xu S, Cecilia Santini G, De Veirman K, Vande Broek I, Leleu X, De Becker A, Van Camp B, Vanderkerken K and Van Riet I: Upregulation of miR-135b is involved in the impaired osteogenic differentiation of mesenchymal stem cells derived from multiple myeloma patients. PLoS One 8: e79752, 2013.

56. Ell B and Kang Y: MicroRNAs as regulators of bone homeostasis and bone metastasis. Bonekey Rep 3: 549, 2014.

57. Zoni E and van der Pluijm G: The role of microRNAs in bone metastasis. J Bone Oncol 5: 104-108, 2016.

58. Li H, Zhang K, Liu LH, Ouyang Y, Guo HB, Zhang H, Bu J and Xiao T: MicroRNA screening identifies circulating microRNAs as potential biomarkers for osteosarcoma. Oncol Lett 10: 1662-1668, 2015

59. Lian F, Cui Y, Zhou C, Gao K and Wu L: Identification of a plasma four-microRNA panel as potential noninvasive biomarker for osteosarcoma. PLoS One 10: e0121499, 2015.

60. Dong J, Liu Y, Liao W, Liu R, Shi P and Wang L: miRNA-223 is a potential diagnostic and prognostic marker for osteosarcoma. J Bone Oncol 5: 74-79, 2016.

61. Ma W, Zhang X, Chai J, Chen P, Ren P and Gong M: Circulating miR-148a is a significant diagnostic and prognostic biomarker for patients with osteosarcoma. Tumour Biol 35: 12467-12472, 2014.

62. Ziyan W, Shuhua Y, Xiufang W and Xiaoyun L: MicroRNA-21 is involved in osteosarcoma cell invasion and migration. Med Oncol 28: 1469-1474, 2011.

63. Ren X, Shen Y, Zheng S, Liu J and Jiang X: miR-21 predicts poor prognosis in patients with osteosarcoma. Br J Biomed Sci 73: 158-162, 2016.

64. Nakka M, Allen-Rhoades W, Li Y, Kelly AJ, Shen J, Taylor AM, Barkauskas DA, Yustein JT, Andrulis IL, Wunder JS, et al: Biomarker significance of plasma and tumor miR-21, miR-221, and miR-106a in osteosarcoma. Oncotarget 27: 96738-96752, 2017.

65. Keremu A, Aini A, Maimaitirexiati Y, Liang Z, Aila P, Xierela P, Tusun A, Moming $\mathrm{H}$ and Yusufu A: Overcoming cisplatin resistance in osteosarcoma through the miR-199a-modulated inhibition of HIF-1 $\alpha$. Biosci Rep: BSR20170080, 2017. 
66. Dalle Carbonare L, Valenti MT, Zanatta M, Donatelli L and Lo Cascio V: Circulating mesenchymal stem cells with abnormal osteogenic differentiation in patients with osteoporosis. Arthritis Rheum 60: 3356-3365, 2009.

67. Weilner S, Skalicky S, Salzer B, Keider V, Wagner M, Hildner F, Gabriel C, Dovjak P, Pietschmann P, Grillari-Voglauer R, et al: Differentially circulating miRNAs after recent osteoporotic fractures can influence osteogenic differentiation. Bone 79: 43-51, 2015.

68. Kocijan R,MuschitzC, GeigerE, Skalicky S,Baierl A, Dormann R, Plachel F, Feichtinger X, Heimel P, Fahrleitner-Pammer A, et al: Circulating microRNA signatures in patients with idiopathic and postmenopausal osteoporosis and fragility fractures. J Clin Endocrinol Metab 101: 4125-4134, 2016.

69. Li H, Wang Z, Fu Q and Zhang J: Plasma miRNA levels correlate with sensitivity to bone mineral density in postmenopausal osteoporosis patients. Biomarkers 19: 553-556, 2014.

70. Seeliger C, Karpinski K, Haug AT, Vester H, Schmitt A, Bauer JS and van Griensven M: Five freely circulating miRNAs and bone tissue miRNAs are associated with osteoporotic fractures. J Bone Miner Res 29: 1718-1728, 2014.

71. Hackl M, Heilmeier U, Weilner S and Grillari J: Circulating microRNAs as novel biomarkers for bone diseases-complex signatures for multifactorial diseases? Mol Cell Endocrinol 432: 83-95, 2016.
72. Yavropoulou MP, Anastasilakis AD, Makras P, Tsalikakis DG, Grammatiki $\mathrm{M}$ and Yovos JG: Expression of microRNAs that regulate bone turnover in the serum of postmenopausal women with low bone mass and vertebral fractures. Eur J Endocrinol 176: 169-176, 2017.

73. Zhang $X$, Li Y, Chen YE, Chen J and Ma PX: Cell-free 3D scaffold with two-stage delivery of miRNA-26a to regenerate critical-sized bone defects. Nat Commun 7: 10376, 2016.

74. Liu J, Dang L, Li D, Liang C, He X, Wu H, Qian A, Yang Z, Au DW, Chiang MW, et al: A delivery system specifically approaching bone resorption surfaces to facilitate therapeutic modulation of microRNAs in osteoclasts. Biomaterials 52: 148-160, 2015.

75. Wright NC, Looker AC, Saag KG, Curtis JR, Delzell ES, Randall S and Dawson-Hughes B: The recent prevalence of osteoporosis and low bone mass in the United States based on bone mineral density at the femoral neck or lumbar spine. J Bone Miner Res 29: 2520-2526, 2014.

76. Zhang J, Tu Q, Bonewald LF, He X, Stein G, Lian J and Chen J: Effects of miR-335-5p in modulating osteogenic differentiation by specifically downregulating Wnt antagonist DKK1. J Bone Miner Res 26: 1953-1963, 2011.

77. Sugatani T and Hruska KA: Impaired micro-RNA pathways diminish osteoclast differentiation and function. J Biol Chem 284: 4667-4678, 2009. 